\title{
Frieden schließen auf Konzilien? Zwei Beispiele aus dem vierten Jahrhundert
}

\author{
Prof. Dr. Thomas Graumann \\ Homerton College, University of Cambridge, Faculty of Divinity \\ tg236@cam.ac.uk
}

\begin{abstract}
The essay examines two exemplary attempts to 'make peace' between rivaling theological factions in the middle of the fourth century. The negotiations conducted in Alexandria in 362, of which the Tomus ad Antiochenos provides a distillation, confirm the importance of exploring theological concepts and terminological preferences on all sides in order to find common ground. Conflicting loyalties ultimately fracture chances for an accord. The so-called second session of Rimini (359) - as presented by Jerome - reveals more clearly the crucial significance of establishing the legitimacy of any agreement in social and cultural as well as in intellectual terms. Former opponents present and perform the theological consensus achieved in a public display so that regained communion can be seen, heard and experienced. In the process the import of theological consent is amplified and transformed by ceremonial enactment into the celebratory demonstration of harmony and communality as well as a common mind.
\end{abstract}

\section{Keywords}

Synod of Alexandria (362) - Synod of Rimini (359) - Schism of Antioch - Constantius II, Roman Emperor (337-361) - Council Acts - Consensus - Creedal formula - Trinitarian Terminology - Conflict resolution - Anathemas

* Dieser Beitrag ist Teil eines Projekts, das durch den Europäischen Forschungsrat (European Research Council, ERC) im Rahmen des EU-Programms für Forschung und Innovation "Horizont 2020" gefördert wurde (Fördervereinbarung Nr. 677638). 
Wenn man an die Synodalgeschichte des 4. Jh. denkt, ist Frieden sicher nicht die erste Assoziation. Im Gegenteil, die Periode ist unter theologiegeschichtlicher Rücksicht ja nicht umsonst traditionell als Arianischer Streit bekannt. In der Auseinandersetzung um die Trinitätstheologie, die ja nicht etwa mit dem Nizänischen Konzil endet, sondern geradezu erst eigentlich beginnt - oder doch wenigstens eine neue, intensivere Dimension annimmt - ist die überwältigende Vielzahl von Bischofsversammlungen konkurrierender Gruppen kennzeichnend. Immer neue Versammlungen versuchen mit neuen Texten und Beschlüssen zu einem adäquaten und sozial sowie politisch vermittelbaren Verständnis der Trinitätstheologie zu gelangen. Dabei kommt es zu heftigen Konflikten und tiefen Spaltungen zwischen verschiedenen Gruppierungen und ganzen Kirchenregionen. Der vielleicht dramatischste Ausdruck dieser Situation ist mit dem Konzil von Serdica (343) verbunden, das faktisch ein Schisma zwischen dem Ost- und Westteil der Reichskirche heraufführt und damit spätere Spaltungen vorwegnimmt. ${ }^{1}$

Nicht erst der moderne Beobachter nimmt im Blick auf die Synodalgeschichte des 4. Jahrhunderts primär Konflikt wahr, auch die Beteiligten selbst betrachten diese Geschehen zunehmend kritisch und geradezu verzweifelt. Basilius von Caesarea etwa spricht von einer Seeschlacht bei Nacht, wo Freund und Feind kaum noch unterscheidbar sind, ${ }^{2}$ und im Blick speziell auf die zahlreichen Synoden, auf denen sich der Streit austrägt, kann sein Kollege Gregor von Nazianz resigniert feststellen: Nichts Gutes kommt je von einer Synode! ${ }^{3}$ Eine Konfliktgeschichte und keine Friedensgeschichte steht uns vor Augen.

Der voranschreitenden Zersplitterung durch konkurrierende Theologien und Loyalitäten steht aber gleichzeitig auch eine Gegenbewegung der Suche nach Übereinkunft und kirchlicher Gemeinschaft entgegen. Das kaiserliche Interesse an einer reichseinheitlichen Kirche befördert solche Tendenzen, ist aber nicht primär ursächlich für sie - und entfaltet zuweilen geradezu kontraproduktive Wirkungen. Vielmehr motiviert das Ideal kirchlicher Einheit

1 Vgl. im weiteren Horizont (und mit nur beiläufigem Hinweis auf das Konzil von Serdika) H. CHADWick, East and West. The Making of a Rift in the Church: From Apostolic Times Until the Council of Florence, Oxford 2003.

2 Bas., Spir. 30,76f. (Basılius von Cäsarea, De Spiritu Sancto/Über den Heiligen Geist, übers. u. eingeleitet v. H.J. Sieben, Freiburg u.a. 1993 [= FC 12], 312-320); vgl. ep. 69,2 (ed. Y. Courtonne, Saint Basile. Lettres. Texte établi et traduit, 3 Bde., Paris 1957-1966 [= CuFr]);

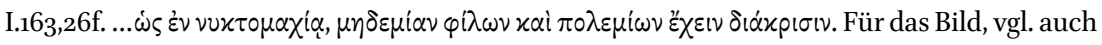
Bas., De Ieunio = Hom. 1 (PG 31, 181D).

3 Greg.Naz., ep. 130,1f. (Gregor von Nazianz, Briefe, hg. v. PAul Gallay, Berlin 1969 [= GCS

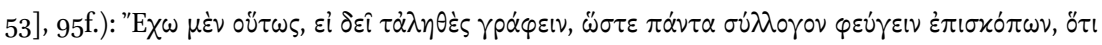

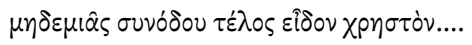


vielfältige Versuche, zueinander zu finden. Einige Gruppen freilich werden dauerhaft marginalisiert und als "Häresien" von diesem Prozess ausgeschlossen. Andere jedoch mühen sich in unterschiedlicher Weise, Differenzen $\mathrm{zu}$ überbrücken und zusammen zu finden. "Frieden" ist dafür ein häufiges Stichwort. Um nochmals Basilius als Beispiel anzuführen, im gleichen Brief,

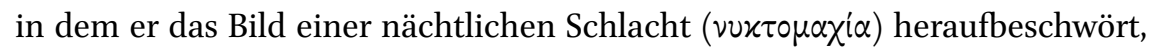
versucht er (vergeblich), die guten Beziehungen des Athanasius nach Rom für seinen Versuch der Friedensstiftung und Wiederherstellung von Gemeinschaft

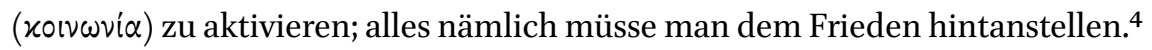
Speziell hat er die Situation rivalisierender Gemeinden in Antiochien im Auge, die uns im folgenden beschäftigen wird.

In der gebotenen Kürze skizziere ich zwei Kontexte, die auf je verschiedene Dimensionen der Friedensbemühungen aufmerksam machen. Die bekannten Umstände beider Zusammenkünfte, um die es mir geht, umreiße ich knapp und vergröbernd nur insoweit, als es für eine Orientierung und zum Verständnis meiner Ausführungen nötig ist. Insbesondere den theologischen Detailfragen, die jeweils zur Sprache kommen, gehe ich nicht nach. ${ }^{5}$

Entgegen der chronologischen Reihenfolge soll uns zunächst die Synode von Alexandria 362 beschäftigen, die für die Situation in Antiochien - die auch bei Basilius im Hintergrund stand - von zentraler Bedeutung ist. Als ein Ergebnis der Zusammenkunft beschreibt der sog. Tomus ad Antiochenos, ${ }^{6}$ ein von Athanasius im Namen der Synode verfasster Brief, die Grundlagen, auf der zwei Gruppierungen in Antiochien die Möglichkeit sondieren sollten, zueinander in Gemeinschaft zu treten. ${ }^{7}$ Vertreter beider Gruppen hatten an der

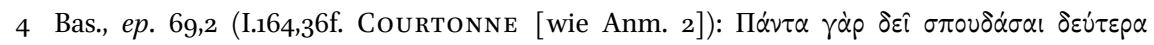

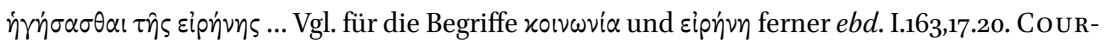
TONNE (wie Anm. 2).

5 Von den Darstellungen der Theologiegeschichte der Mitte des 4. Jahrhunderts, s. exemplarisch R.P.C. HAnson, The Search for the Christian Doctrine of God. The Arian Controversy, 318-381, Edinburgh 1988. L. Ayres, Nicaea and its Legacy: An Approach to Fourth-Century Trinitarian Theology, Oxford 2004. Zur theologischen Einordung spezifisch des Tomus vgl. Anm. 8.

6 Tomus ad Antiochenos, in: Athanasius Werke Bd. II: Die "Apologien”, Lieferung 8, hg. von H.C. Brennecke/U. Heil/A.v. Stockhausen, Berlin 2006, 341-351. Der Tomus wird im Folgenden nach dieser Ausgabe unter Angabe der Dokumenten-Nummer zitiert. Der Tomus ist wieder abgedruckt in: Athanasius Werke III.1: Dokumente zur Geschichte des Arianischen Streits, 4. Lieferung: Bis zur Synode von Alexandrien 362, hg. von H.C. Brennecke/A. v. Stockhausen/C. Müller/U. Heil/A. Wintjes, Berlin 2014, 592-6o3 (Doc. 69.2).

7 Zu den geschichtlichen Umständen der Synode, vgl. knapp die Einführung Athanasius Werke III.1, 589f. und die historische Kommentierung Doc. 69.2 (s. vorige Anm.). Aus der reichen 
Synode teilgenommen, und der Brief skizziert, was dort verhandelt worden war und welche Übereinkunft man erreicht hatte. Mehrfach ist im Brief vom Frieden als dem Ziel die Rede, das beide Gruppen anstreben sollten (

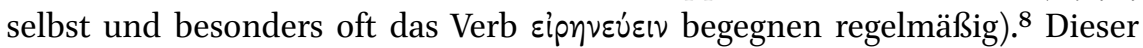
Friede, das ist ebenfalls klar, besteht in der Aufnahme der Kirchengemeinschaft $\left(\varkappa_{1} v \omega v^{\prime} \alpha\right)^{9}-$ der Gegenbegriff ist Schisma $\left(\sigma \chi^{i} \sigma \mu \alpha\right) .{ }^{10}$ Der angestrebte Friedenszustand gründet sich auf und beinhaltet soziale ebenso wie theologische Übereinstimmung. Zum einen wird die Übereinstimmung im Denken angezielt, die man im Konkreten als theologischen Konsens verstehen muß, ${ }^{11}$ zum anderen werden mit Harmonie und Eintracht soziale Kategorien evoziert. ${ }^{12}$ Diese Dimension wird besonders durch das aufgerufene Gegenbild von

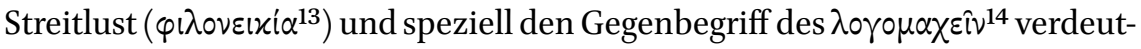
licht: Bedingung und Kennzeichen des auch sozial verwirklichten Kirchenfriedens ist der Verzicht auf weiteren Streit um Worte. Theologisch gemeint ist

Literatur, zumal bezüglich der theologischen Einordnung, vgl. M. TETz, Über nikäische Orthodoxie. Der sog. Tomus ad Antiochenos des Athanasios von Alexandrien, in: ZNW 66 (1975) 194-222 (= DERS., Athanasiana. Zu Leben und Lehre des Athanasius, hg. v. W. Geerlings/D. Wywra, Berlin 1995 [= BZNW 78], 107-134); C. Yeum, Die Synode von Alexandrien (362). Die dogmengeschichtliche und kirchenpolitische Bedeutung für die Kirche im 4. Jahrhundert, Münster u.a. 2005 (= Studien zur Orientalischen Kirchengeschichte 34); P. Gemeinhardt, Der Tomus ad Antiochenos (362) und die Vielfalt orthodoxer Theologien im 4. Jahrhundert, in: ZKG 117 (2006) 169-196. Dass die Synode tatsächlich eine Versöhnung in Antiochien zu befördern suchte - und damit hier zur Friedensthematik einschlägig ist -, hat gegen Zweifel jüngst nochmals affirmiert D. Fairbairn, The Sardican Paper, Antiochene Politics, and the Council of Alexandria (362): Developing the "Faith of Nicaea", in: JThS 66 (2015) 651-678, hier 666-674; s. ferner M. Mira, El sínodo de Alejandría del 362 y la pacificación de la Iglesia antioquena in diesem Band.

8 Für eipín一 s. Tom. 2,1 (342,6 Brennecke/Heil/v. Stockhausen [wie Anm. 6]); 8,2

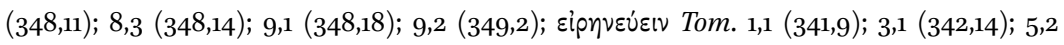
(344,10f.).

9 Tom. 1,1 (341,11 Brennecke/Heil/v. Stockhausen [wie Anm. 6]); 4 (343,12f.). Der Einheitsgedanke, im Denken wie in der Kirchengemeinschaft, ist zugleich emphatisch durch den Ruf ein Herr, ein Glaube (Eph 4,4 zitiert Tom. 1,1 [341,16]) grundgelegt.

10 Tom. 8,2 (348,12 Brennecke/Heil/v. Stockhausen [wie Anm. 6]).

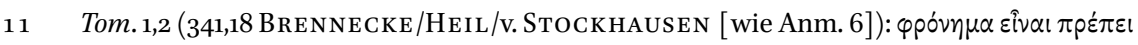

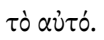

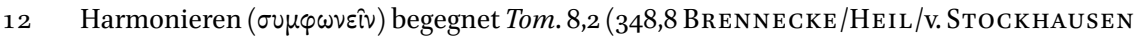
[wie Anm. 6]); Eintracht (óróvoเ $\alpha$ ) Tom. 2,1 (342,6); 4,1 (343,17).

13 Tom. 1,1 (341,9 Brennecke/Heil/v. Stockhausen [wie Anm. 6]); 5,2 (344,9. 14); 9,1

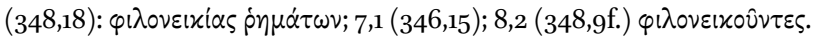

14 Tom. 8,2 (348,7 Brennecke/Heil/v. Stockhausen [wie Anm. 6], als Zitat von 2 Tim 2,14); vgl. 348,8: $\lambda \dot{\varepsilon} \xi \xi \sigma \iota ~ \delta 1 \alpha \mu \alpha ́ \alpha \varepsilon \sigma \theta \alpha$. 
konkret die Debatte um den bisher trennenden Sprachgebrauch trinitarischer Ousia- und Hypostasenlehre. Daneben aber wird eine schon topische Abneigung gegen den streitigen Austrag solcher Differenzen, oft assoziiert mit einem falschen Gebrauch der Dialektik, angespielt. ${ }^{15}$ Solches Streiten um Worte ist klischeehaftes Charakteristikum für die Häresie. In einer auf gemeinsamer Rechtgläubigkeit aufruhenden und sie verwirklichenden Friedenssituation hat sie keinen Platz.

Entsprechend soll Frieden zwischen den antiochenischen Parteien möglich werden auf der Grundlage einer Übereinkunft über die theologische Terminologie und die zugehörigen Konzeptionen bezüglich der Rede von einer Hypostase oder drei Hypostasen der Trinität. Der Tomus berichtet, wie die Vertreter des je anderen Sprachgebrauchs daraufhin befragt wurden, ob ihre Rede bestimmte, als häretische empfundene (Miss-)verständnisse beinhalte oder gar lehre. Die Befragten konnten der je anderen Seite klarstellen, was in ihren Augen der positive Sinn ihrer bevorzugten Terminologie war, und wie sie keinesfalls das von den anderen Befürchtete vertraten. ${ }^{16}$ Damit, und unter der zwischen ihnen infolgedessen unproblematisch gewordenen Bedingung erstens der Anerkennung des Nizänums und zweitens der Gottheit des Geistes, würde man Einigung erzielen können. ${ }^{17}$

In Antiochien scheiterte allerdings der Einigungsversuch, nicht zuletzt, weil inzwischen mit Paulinus dort auch ein Vertreter der Altnizäner zum Bischof geweiht worden war und damit ein lang anhaltendes Schisma zweier nizänischer Gruppen festgeschrieben wurde. ${ }^{18}$

Für unser Interesse gilt es festzuhalten, dass der angestrebte Friede im Kern auf der Basis einer theologischen Klarstellung erfolgen sollte und sich auf Akzeptanz und Ablehnung bestimmter Formulierungen stützt. Friede ist Ergebnis und Ausdruck theologischer Übereinstimmung, im konkreten Fall freilich anfangs so, dass man willens war, unterschiedlichen Sprachgebrauch zu tolerieren, nachdem man sich überzeugt hatte, dass damit nichts "Häretisches" gemeint war. Diese Form der Friedensfindung ist wohl das, was zumal der Theologiegeschichtler erwartet: Versöhnung über theologische

15 Vgl. R. Lim, Public Disputation, Power, and Social Order in Late Antiquity, Berkeley 1995 (= The transformation of the classical heritage 23).

16 Vgl. Tom. 5,3-6,4 (344-346 Brennecke/Heil/v. Stockhausen [wie Anm. 6]). Vgl. jüngst T.R. Karmann, Meletius von Antiochien: Studien zur Geschichte des trinitätstheologischen Streits in den Jahren 360-364 n. Chr., Frankfurt u.a. 2006 (= RSTh 68), mit ausführlicher Diskussion des Tomus 193-305; YeUM (wie Anm. 7).

17 Tom. 3,1-3.

18 Klassisch F. Cavallera, Le Schisme d'Antioche (IVe-Ve siècle), Paris 1905. Ausführlich zur Ordination des Paulinus, vgl. ferner Karmann (wie Anm. 16), 306-321. 
Differenzen wird hergestellt im Modus der theologischen Reflexion und Sachklärung, hier der Begriffsklärung. Die theologische Spaltung wird geheilt durch besseres theologischen Nachdenken, Argumentieren und Formulieren, sowie in der Anerkenntnis einer gemeinsamen Bekenntnisbasis. Das korrespondierende Bild einer Synode (gleich welchen institutionellen Status man nun der Zusammenkunft in Alexandria zuschreiben möchte) als Ort und Instrument theologischer Reflexion und Definition, wohl auch Verhandlung, fügt sich in ein theologiegeschichtlich orientiertes Deutungsschema und entspricht den oft unreflektierten - Erwartungen an die Synode als ein primär theologisch motiviertes Forum (sofern denn nicht disziplinarische Probleme zur Verhandlung kommen). Aber die im Tomus ad Antiochenos umrissene Versöhnung wird nicht gesellschaftliche und kirchliche Realität. Die Zusammenführung beider Gruppen in Antiochien scheitert. Stattdessen begründet die Weihe des Paulinus zum Bischof der Altnizäner ein andauerndes Schisma.

Ein zweites Beispiel aus der Mitte des Jahrhunderts verdeutlicht denn auch, dass mit der Suche nach theologischer Klarstellung noch nicht das Ganze der kirchlichen und synodalen Friedensstiftung im Blick ist. Das Scheitern der Übereinkunft in Antiochien über Personalfragen und Loyalitäten deutet schon an, dass mit rein intellektueller theologischer Friedensbemühung noch nicht alles gewonnen ist, und eine "bessere Theologie" und geklärte Fachterminologie nicht notwendigerweise hinreichend sind, um dauerhaften Frieden zu gewährleisten. Selbst im Trinitarischen Streit geht es nicht allein um "Wahrheit", sondern auch um gesellschaftlich-kirchlichen Konsens und die Herstellung von Legitimität. Anders gesagt, das sachlich Gewonnene muss auch gesellschaftlich-kirchlich vermittelbar sein und sich als legitim bzw. verbindlich ausweisen können, um wirksam zu werden. Dazu gehört dann allerdings auch noch die politische Durchsetzbarkeit, wie gerade die kaiserlichen Eingriffe in die theologischen Streitigkeiten des 4. und 5. Jh. vielfältig illustrieren. Diese Thematik will ich hier jedoch nur andeuten und nicht weiter verfolgen.

Stattdessen geht mein zweites Beispiel der Spur nach, wie sachliche Übereinstimmung kirchlich-gesellschaftlich darstellbar werden konnte oder musste. Es betrifft das Geflecht der miteinander verwobenen Synoden von Rimini, Seleukia und Konstantinopel in den Jahren 359-6o. So sehr die kaiserliche Religionspolitik für die Choreographie der Ereignisse und die Durchsetzung bestimmter theologischer Formeln einer der bestimmenden Faktoren ist, soll hier vielmehr ausgeleuchtet werden, wie kirchliche Akteure eine durchaus strittige und als problematisch empfundene Übereinkunft vor der kirchlichen Öffentlichkeit zu legitimieren versuchten. Ein sachlicher Zusammenhang mit der zeitlich späteren, soeben knapp besprochenen Synode von Alexandria, besteht dabei insoweit, als das Treffen in Alexandria sich an dem offiziellen 
Ergebnis jener Synoden abarbeitet und versucht, sie auszuhebeln oder unwirksam zu machen.

Das Doppel-Konzil von Ariminum (Rimini) und Seleukia sollte die entscheidende Weichenstellung in der Einigungspolitik des Kaisers Konstantius II. darstellen, der seit seiner Alleinherrschaft 351, wie sein Vater Konstantin vor ihm, konsequent nach einer einheitlichen Reichstheologie strebte und allen Widerstand zu überwinden, notfalls zu brechen, gewillt war. Konstantius lud zu zwei separaten Synoden der Bischöfe beider Reichsteile, verlangte, dass sie ihre Theologie schriftlich niederlegten - eine früher im Jahr zusammengekommene Vorbereitungsgruppe hatte einen Entwurf vorbereitet ${ }^{19}$-; sodann sollten beide Seiten Delegationen von je zehn Bischöfen zum Hof schicken, die dort die endgültige Einigung aushandeln und formalisieren sollten, die dann wiederum zurück vor die jeweilige Synode gebracht und ihr zur Unterschrift vorgelegt werden sollte. ${ }^{20}$ Seine entsprechenden Anweisungen legen präzise die erwarteten Schritte, die Aufgabe der Synode(n) und die Grenze ihrer Kompetenzen fest. ${ }^{21}$ So entsteht eine komplizierte Choreographie von synodaler Diskussion, diplomatischer Mission und Verhandlung am Hof, die wiederum zurückmünden sollte in eine synodale Inkraftsetzung des erzielten Konsenses. Die geplante Sequenz von Treffen und die Befassung mit theologischen Positionspapieren klingt zunächst ganz so, als solle auch hier auf diskursivem Wege in kühler Rationalität der Versuch einer theologischen Sachklärung betrieben werden, und als werde sodann das gewünschte Resultat vor allem durch kaiserliche Machtausübung gewährleistet.

19 Die kleine Gruppe von Bischöfen entwarf in Sirmium das von den Gegnern vielfach verspottete sog. Datierte Bekenntnis (22. Mai 359), überliefert bei Athan., syn. 8,3-7 (235,21236,15 OpITz); Socr., h.e. 2,37,18-24 (154,6-155,9 HANSEN). Vgl. zur Theologie der Formel W.A. LöHR, Die Entstehung der homöischen und homöusianischen Kirchenparteien. Studien zur Synodalgeschichte des 4. Jahrhunderts, Bonn 1986 (= BBKT 2), 99-102; H.C. BRENNECKE, Studien zur Geschichte der Homöer. Der Osten bis zum Ende der homöischen Reichskirche, Tübingen 1988 (= BHTh 73), 16-23.

20 Ausführlich und grundlegend für den gesamten hier ins Auge gefassten Abschnitt kaiserlicher und kirchlicher Einigungsbemühungen, s. BREnNECKE, Studien zur Geschichte der Homöer (wie Anm. 19), 5-46; LöHR (wie Anm. 19), 103-129. Vgl. zur Geschichte der Synode ferner C.J. HEFELE und H. LECLERQ, Histoire des conciles d'après les documents originaux, 11 Bde., (Paris 1907-1952) ND Hildesheim 1973, Bd. I/2 (1907=1973), 929-955, M. SimonetTi, La crisi ariana nel IV secolo, Rom 1975 (= SE Aug 11), 314-325; T.D. BARnes, Athanasius and Constantius. Theology and Politics in the Constantinian Empire, Cambridge - Mass. u.a. 1993, 145-149; sowie die Rekonstruktion der komplexen Geschichte der Vorbereitung der Synode bei LöHR (wie Anm. 19), 93-98.

21 Hil., coll. antiar. A 8 (Collectanea antiariana Parisina, ed. A. FEder, Wien 1916 [= CSEL 65], 93f.). 
Wenn wir im folgenden des näheren speziell auf die sog. zweite Sitzung des Konzils von Rimini schauen, zeigt sich jedoch, dass einzelne Aktivitäten der Synode aus den Bedürfnissen und Notwendigkeiten theologischer Sachdebatte nicht hinreichend erklärt werden können. Vielmehr wird uns ein Konzilsgeschehen vor Augen geführt, das als hochgradig inszeniert begriffen werden muss und - mindestens in Teilen - rituell-symbolischen Charakter hat. Den bischöflichen Aktivitäten kommt die Aufgabe zu, nicht erst eine thematisch-sachliche Übereinstimmung herbeizuführen, sondern einen bereits mit anderen Methoden erzielten Konsens im Blick auf eine weitere Öffentlichkeit darzustellen und auszuagieren. Gerade bei dem Versuch, Versöhnung und Frieden herbeizuführen, ist die theologische Formulierungskunst allein nicht ausreichend, und genügt es auch nicht, rein machtpolitisch zu agieren. Das inszenierte symbolische Interagieren der Bischöfe überbrückt die Lücke zwischen sachlicher theologischer Übereinstimmung und seiner gesellschaftlichkirchlichen Realisierung; es schafft zugleich das Vertrauen in die Integrität der Handelnden und ihre Motive angesichts machtpolitischer Verdachtsmomente.

Zunächst allerdings scheiterte das von Konstantius so kunstvoll arrangierte Verfahren. Zumal die westliche Synode lehnte mehrheitlich den vorgelegten theologischen Entwurf ab und verdammte dessen Sponsoren, die Bischöfe Valens von Mursa (Osijek) und Ursacius von Singidunum (Belgrad). ${ }^{22}$ Die Synode teilte sich, und beide Seiten sandten Delegationen zum Hof. Doch statt zum Hof nach Konstantinopel vorgelassen zu werden, tagten die Gruppen zunächst im thrakischen Nike. Die dortige Druckkulisse - die genauen weiteren Gründe bleiben letztendlich im Dunkeln - trug dazu bei, dass die Delegation der hier behandelten pro-nizänischen westlichen Teilsynode die Verurteilung des Valens und seiner Genossen schließlich aufhob, Gemeinschaft mit ihnen

22 Für die in den Quellen stets im Gespann auftretenden Bischöfe vgl. J. ULRICH, Ursacius von Singidunum und Valens von Mursa, in: $\mathrm{RGG}^{4}, 8$ (2005) 837-838; (J. M. SimonetTi), Valens of Mursa and Ursacius of Singidunum, in: Encyclopedia of the Early Church, ed. A. di Berardino (transl. A. WAlford), 2 Bde., Cambridge 1992, hier II 858; B. WindaU, Art. Valens von Mursa, in: LACL ${ }^{2}$, 62of. Nochmalige ausführliche Darlegung ihrer Aktivitäten in den 350er Jahren in kirchenpolitischer Hinsicht, dabei aber kritisch zu ihrer konventionellen Beschreibung als "Hofbischöfe", s. jetzt auch P. JusT, Imperator et Episcopus. Zum Verhältnis von Staatsgewalt und christlicher Kirche zwischen dem 1. Konzil von Nicaea (325) und dem 1. Konzil von Konstantinopel (381), Wiesbaden 2003 (= Potsdamer Altertumswissenschaftliche Beiträge 8), 73-77. Die in der traditionellen Bezeichnung zugleich angespielte Deutung der Ereignisse als Ausfluss vorrangig kaiserlich-politischer Einflussnahme (vgl. aber die oben, Anm. 7 und 16 genannte Literatur) ist in der hier versuchten Analyse der Darstellungsweisen des Friedensschlusses weder vorausgesetzt, noch wäre sie von ihr außer Kraft gesetzt. 
aufnahm und sogar eine nur geringfügig modifizierte Version des zuvor verworfenen Texts unterschrieb. ${ }^{23}$ Als die Delegation nach Rimini zurückkehrte, weigerte sich die Synode zunächst, die Beteiligten überhaupt zu empfangen, da sie völlig konträr zu ihrem Mandat gehandelt hatten. Erst nach beträchtlichem Widerstand und nicht ohne abermaligen staatlichen Druck kam es zu einer Übereinkunft. In einem obskuren Manöver, das schon Zeitgenossen als den Betrug von Rimini bezeichneten, wurde Einvernehmen erzielt. ${ }^{24}$

Um diese schließlich erreichte Versöhnung soll es hier gehen. In der Perspektive unserer Tagung nämlich kann sie als Friedensschluss beschrieben werden. Hieronymus, dem wir den Bericht und die Dokumente verdanken, bezeichnet allerdings, das muss festgehalten werden, das Erreichte gerade nicht als Frieden. Wir dürfen wohl annehmen, dass dies hauptsächlich damit zu tun hat, dass Hieronymus das Synodalergebnis als gravierende Verfehlung einschätzt. Für ihn ist es gleichbedeutend mit der Anerkenntnis des Arianismus. Sein Fazit ist das vielzitierte Bonmot: Ingemuit totus orbis, et Arianum se esse miratus est (Der ganze Erdkreis stöhnte und wunderte sich, dass er arianisch war). ${ }^{25}$

Betrachten wir jedoch die Abläufe, mit deren Hilfe Frieden wiederhergestellt wurde. Auch hier begegnen zunächst diejenigen Elemente, die wir schon zuvor beim Tomus ad Antiochenos beobachtet hatten: Frieden bedeutet Kirchengemeinschaft; Grundlage ist die Akzeptanz bestimmter Bekenntnisformulierungen, und auch hier müssen der Häresie verdächtige Einzelne sich von bestimmten Anschauungen und Begriffen distanzieren. Das geschieht in

23 Bei dem fraglichen Text handelt es sich um den Beschluss von Nike; er ist überliefert durch Hilarius, coll.antiar. A 5,3 (85f. Feder [wie Anm. 21]), die Formel findet sich auch bei Thdt., h.e. 2,21,3-7 (L. PARMentier/F. Scheidweiler, Theodoret, Kirchengeschichte, 3., durchges. Aufl. von G.C. Hansen, Berlin 1998 [= GCS 44], 145,4-146,12); zu den Umständen vgl. Brennecke, Studien zur Geschichte der Homöer (wie Anm. 19), 33f.

24 Y.M. Duval, La «manœuvre frauduleuse» de Rimini: à la recherché du Liber adversus Vrsacium et Valentem, in: Hilaire et son temps. Actes du Colloque de Poitiers 29 Septembre -3 Octobre 1968, Paris 1969, 51-103, repr. in: ID., L'extirpation de l'Arianisme en Italie du nord et en occident: Rimini (359/6o) et Aquilee (381), Hilaire de Poitiers ( $† 367 / 8)$ et Ambroise de Milan († 397), Brookfield, 1999 (=Variorum Collected Studies Series 611); vgl. ferner Brennecke, Studien zur Geschichte der Homöer (wie Anm. 19), 38f., LöHr (wie Anm. 19), 113-116.

25 Hier., c. Lucif. 19 (Hieronymus, Altercatio Luciferiani et Orthodoxi, ed. A. Canellis, Turnhout 2000 [= CCSL 79B], 48,688); zugleich: Jérôme, Débat entre un Luciférien et un Orthodoxe (Altercatio Luciferiani et Orthodoxi), introd., texte critique, trad., notes et index par A. CANE LLIS, Paris 2003 (= SC 437), 158,10f. Die Altercatio wird nachfolgend nach der Ausgabe SC 437 zitiert. 
unserem Fall in aller Form, durch Anathematismen (dazu unten). Es kommt aber etwas anderes hinzu.

Um den Strategien und Bedürfnissen der Herstellung von Frieden auf die Spur zu kommen, müssen wir den Bericht der Ereignisse näher untersuchen. Über die fraglichen, konventionell als zweite Sitzung von Rimini apostrophierten Vorgänge berichtet Hieronymus in der sog. Altercatio mit den Luciferanern. ${ }^{26}$ Dafür dass diese Einigung möglich wurde, trug nach seinem Bericht entscheidend bei, dass Valens sich bereit fand, arianische "Blasphemien" zu verdammen. Anscheinend gab es Gerüchte, dass die zuvor unterzeichnete Formel irgendeine Verschleierung der in Wahrheit arianischen Denkweise enthalte Hieronymus spricht von Betrug: fraus. Darum distanzierte sich Valens förmlich von Arius und drückte seine Abscheu vor arianischen Überzeugungen aus, um sich von solchen Verdächtigungen zu reinigen. ${ }^{27}$ Die Möglichkeit, einen theologischen Text zu akzeptieren, verlangt also nicht allein dessen intellektuelle Valenz, sondern auch persönliches Vertrauen in seinen Verfechter (und Mitautor). Nicht ausschließlich diese oder jene theologische Begrifflichkeit und Argumentation, sondern erst ein zusätzliches persönliches Dementi der Anhängerschaft von dem eponymen Vater der Häresie, (womöglich schon hier als Anathema?), reinigt vom Verdacht. Dem Theologiehistoriker ist vertraut, dass diese eher grobschlächtige Abweisung des Arius den fortgeschrittenen Diskussionsstand in der Sache gerade nicht adäquat aufgreift; "Arius" ist hier längst polemische Chiffre - und aus der Gegnerperspektive des Hieronymus sogar häresiologisches Etikett - einer als homöisch zu beschreibenden Auffassung. Die Abweisung des "Arius" ist gleichwohl kaum erst dem kritischen Bericht des Nizäners Hieronymus wichtig. Die Verkürzung der Positionsbestimmung auf ein antihäretisches Schlagwort und Zurückweisung seiner Symbolfigur mochte vielmehr durchaus geeignet sein, das Misstrauen der beteiligten Bischöfe gegenüber Valens - und womöglich mehr noch das einer weiteren Öffentlichkeit zu beruhigen. Allein da Valens seine Versicherung zunächst res secrete gesta abgab, kam die Gerüchteküche nicht zum Stillstand und wurde die Unruhe in der Öffentlichkeit gerade nicht beseitigt. ${ }^{28}$

26 Hier., c. Lucif. 17-18; (148,1-156,53 CANELlis [wie Anm. 26]). Knappe Analyse, ohne Bezug auf die hier untersuchten Verhaltensweisen, bei LöHr, Die Entstehung (wie Anm. 19), $117-129$.

27 Hier., c. Lucif. 18; (150,5-152,10 CANELlis [wie Anm. 25]): Denique, ipso in tempore, cum fraudem fuisse in expositione rumor populi uentilaret, Valens, Mursensis episcopus, qui eam conscripserat, praesente Tauro, praetorii praefecto, qui ex iussu regis synodo aderat, professus est se Arianum non esse et penitus ab eorum blasphemiis abhorrere.

28 Hier., c. Lucif. 18 (152,10 CANELLIS [wie Anm. 25]): Res secrete gesta opinionem uulgi non extinxerat. 
Die kritisierte Heimlichkeit der Erklärung des Valens ist denn auch erstaunlich und befremdlich. Sie kontrastiert mit der Beschreibung des seinerzeitigen ersten Versuchs seiner Gruppe auf der sog. ersten Sitzung der Synode von Rimini, den ursprünglichen Textentwurf vorzustellen und zu empfehlen. Dies war explizit als öffentlich, und zwar in offener und formeller Konzilssitzung (publice in concilio) stattfindend beschrieben. ${ }^{29}$ Demgegenüber erfolgte Valens' Versicherung nunmehr nicht in einer Plenarsitzung der Synode, sondern hinter verschlossenen Türen. Der Bericht des Hieronymus erwähnt die Anwesenheit des Prätoriumspräfekten Taurus. ${ }^{30}$ Es ließe sich konjizieren, dass Valens seine Erklärung vor dem Präfekten und in der Gegenwart nur einiger weniger anderer, etwa während einer Audienz, abgab. Der Präfekt dürfte durchaus konventionell für kaiserliche Beamte wichtige Amtsgeschäfte in einem separierten Raum abgehalten haben, der oftmals durch einen Vorhang vom Rest einer größeren Audienzhalle abgeteilt war. Womöglich erklärt ein solches Arrangement die Beschreibung der Erklärung des Valens als secrete gesta im Sinne einer durchaus üblichen Begrenzung des Zugangs der Öffentlichkeit zu wichtigen juristisch-administrativen Amtsgeschäften besser als die Annahme konspiratorischer Heimlichkeit, deren Funktion und Nutzen fraglich bleiben müsste.

In jedem Fall bleiben die wahrgenommene Unzulänglichkeit dieser Vorgehensweise und das Bedürfnis festzuhalten, die erzielte Einigung und schon fast demonstrative Selbstreinigung vom Arianismus-Vorwurf über den engeren Kreis der kirchlichen und imperialen Amtsträger hinaus vor einer größeren Öffentlichkeit zu demonstrieren und zu inszenieren. Schon bis zu diesem Punkt beinhaltet die Übereinkunft somit zwei Komponenten: die Ablehnung von "Blasphemien", also eine wohl satzhafte, propositionelle Abkehr von theologischen Aussagen und Formel (die nachfolgend abermals begegnen wird); und die Distanzierung von Arius - bzw. dem Arianer-Namen, also eine eher symbolhafte Selbstbeschreibung und abgrenzende Definition der eigenen Identität. Ein zentrales Element wurde gleichwohl noch vermisst. Die Einigung in der Sache konnte nur auf kirchliche und gesellschaftliche Akzeptanz hoffen und so Frieden stiften, wenn darüber hinaus das Vertrauensverhältnis zwischen den Hauptakteuren auch allenthalben sichtbar wiederhergestellt wurde und

29 Diese Einzelheit berichtet der Synodalbrief der Synode an Kaiser Konstantius II; Hil., coll. antiar. A 5.2.3 (82,6 FEDER [wie Anm. 21]): ...quod offerebant publice in concilio ... Man könnte geneigt sein, hierin zugleich einen wichtigen Unterschied zum Tagen jener kleineren Vorbereitungsgruppe ausgedrückt zu sehen, die zuvor den problematischen Text entworfen hatte.

Siehe PLRE 1, 879-88o, s.v. Flavius Taurus. 
wenn insbesondere die Vertrauenswürdigkeit des Valens einer breiten Öffentlichkeit demonstrativ vorgeführt und ihr gegenüber bestätigt wurde. ${ }^{31}$ In ein Verhältnis sowohl sachlicher, d.h. theologischer, als auch kirchlich-politischer Übereinstimmung mit der Synode konnte Valens nur eintreten durch die Herstellung und Darstellung seiner sozialen Integration in den Kreis der legitimen kirchlichen Entscheidungsträger und der durch sie repräsentierten umfassenden kirchlichen (Heils-) Gemeinschaft. Damit ging, wie sich zeigt, zugleich notwendig eine Umdeutung seiner persönlichen Rolle und seines Status im Gegenüber zu beiden einher. Der theologische Konsens mit der Aussicht auf Friedensstiftung wird gesellschaftlich wirksam im Vollzug seiner öffentlichen Darstellung und des handelnd, im Modus liturgisch gelebter und zelebrierter Kirchengemeinschaft, ausgedrückten Beweises der charakterlich-moralischen Festigkeit und Verlässlichkeit der wichtigsten Akteure und damit ihrer Befähigung, überhaupt mit dem Anspruch von Legitimität und Autorität ein solches Übereinkommen einzugehen. Der Friedensschluss wird nicht (mehr) vorrangig diskursiv, sondern performativ geübt.

Darum fand an einem der folgenden Tage eine erstaunliche Vorstellung statt. Das Geschehen darf mit Bedacht als "Vorstellung" apostrophiert werden, da es überdeutliche Zeichen von Inszenierung aufweist. Auch geht es bei der fraglichen Zusammenkunft nicht darum, eine Entscheidung zu erzielen - dies war in der Sache bei dem Treffen vor dem Prätoriumspräfekten bereits geschehen -, sondern sie öffentlich auszuagieren mit dem Ziel, das Bild der Einheit zwischen den Bischöfen und ihres völligen Übereinkommens über die Fragen des Glaubens auszumalen und vorzuführen.

Eine große Menge, Bischöfe und Laien, versammelten sich in der Kirche. ${ }^{32}$ Damit sind zwei bedeutsame Elemente angezeigt, die das nachfolgende Geschehen von den vorherigen Verhandlungen absetzen und in einen veränderten Rahmen einzeichnen: Zum einen wechselt die Situierung in den Raum der Kirche und zum anderen wird ausdrücklich die Präsenz einer weiteren Öffentlichkeit über den Kreis der Bischöfe hinaus festgehalten. Schon damit

$31 \quad$ Für den Zusammenhang zwischen expressivem Handeln und der Herstellung und Erhaltung von Vertrauen, vgl. N. Luhmann, Vertrauen. Ein Mechanismus der Reduktion sozialer Komplexität, Stuttgart ${ }^{4} 2000$ (1. Aufl. Frankfurt/M. 1968). Luhmann unterstreicht die zentrale Bedeutung des Vertrauensproblems bzw. der Beziehungsaspekte für die Ermöglichung sozialen Handelns. Vgl. für diese Dimension auch das Konzept des "sozialen Kapitals" klassisch beschrieben in P. Bourdieu, Ökonomisches Kapital - Kulturelles Kapital - Soziales Kapital, in: R. Kreckel (Hg.), Soziale Ungleichheiten, Göttingen 1983, 183-198.

32 Hier., c. Lucif. 18 (152,11-12 CANELLIS [wie Anm. 25]): Itaque, alia die, in ecclesiam quae est apud Ariminum et episcoporum simul et laicorum turbis concurrentibus.... 
ist klar, dass hier einerseits keine "reichspolitische" Veranstaltung (unter dem Einfluss und in Gegenwart des Präfekten), sondern eine dezidiert "kirchliche" abgehalten wird. Andererseits steht auch keine interne Exploration der Möglichkeit von Konsens oder der Prüfung theologischer Formeln an, sondern gezielt soll das (herzustellende) einvernehmliche Verhältnis zwischen den Bischöfen zum Gegenstand öffentlicher Anschauung werden.

Ein Bischof namens Muzonius aus der Provinz Byzacena, ansonsten keiner der führenden Protagonisten der theologischen und kirchenpolitischen Auseinandersetzungen, ${ }^{33}$ eröffnet das Prozedere; man hatte ihn dazu aufgrund seine Alters eingeladen. ${ }^{34}$ Auch diese Wahl zeigt an, dass in dem nun eingeleiteten Verfahren konventionelle Statusmerkmale, nicht theologisches Fachwissen unterscheidend wirksam werden. Sein etwas unscharfer Verfahrensvorschlag ist, die Dinge, die in der Öffentlichkeit kursierten und unsere Synode erreicht haben zu verlesen, damit was falsch sei verdammt werden könne. ${ }^{35}$ Als die Synode diesen Verfahrensvorschlag aufgreift, beginnt ein gewisser Bischof Claudius ${ }^{36}$ aus einem Text vorzulesen, der angeblich von Valens verfasst sei. ${ }^{37}$ Die Textverlesung dient in merklicher Inszenierung von vornherein nur als Anlass für das nachfolgende, eigentlich angezielte Geschehen; die Reaktionen sind keinesfalls spontan. Valens unterbricht die Verlesung laut rufend; er bestreitet, erstens, seine Autorschaft und spricht, zweitens, das Anathema über eine bestimmte Vorstellung aus, die auf eine im soeben verlesenen Text formulierte Ansicht rekurrieren dürfte: Wenn einer leugnet, dass Christus der Herr,

33 S.A. Mandouze, Prosopographie chrétienne du Bas-Empire, Bd. I: Afrique (303-533), Paris 1982, 768 s.v.; cf. CANELLIS (wie Anm. 25), Altercatio SC 437, 153 ad locum.

34 Hier., c. Lucif. 18 (152,13-14 CANELLIS [wie Anm. 25]): Mizonius, episcopus prouinciae Byzacenae, cui propter aetatem primatus ab omnibus deferebatur, ita locutus est ... Zur Textgestalt, s. CANELLIS (wie Anm. 25), CCSL 79B, p. 82 note critique ad 18,639.

35 Hier., c. Lucif. 18 (152,15-18 CANELLIS [wie Anm. 25]): Ea quae sunt iactata per publicum, et ad nos usque perlata, aliquem e nobis Sanctitati Vestrae legere praecipimus ut, quia sunt mala et ab auribus et a corde uestro abhorrere debent, una omnium uoce damnentur.

$3^{6}$ Bischof der Provinz Pecenum in Italien, auch er keiner der Parteiführer und bestimmenden Theologen der Zeit. Vgl. Ch. Piétri/L. Piétri, Prosopographie chrétienne du Bas Empire. II: Italie (313-604), 2 Bde., Paris 1999, hier I 448 s.v. Claudius 1.

37 Hier., c. Lucif. 18 (152,20-22 CANE LLIS [wie Anm. 25]): Itaque, cum Claudius, episcopus prouinciae Piceni, ex praecepto omnium, blasphemias quae Valentis ferebantur legere coepisset ... Einen eigenständigen "Text" mit den fraglichen Blasphemien zu identifizieren ist mir nicht gelungen; das wenige, was von Valens erhalten ist oder ihm zugeschrieben wird, besteht aus Briefen und beinhaltet keinen theologischen Traktat oder ähnliches Material; vgl. CPL 682-687. Sollte es sich bei den verlesenen Blasphemien schlicht um eine thesenhafte Form von Spitzenaussagen handeln, die sodann unmittelbar ins Negative gekehrt als Anathematismen präsentiert wurden? 
der Sohn Gottes vor den Zeiten aus dem Vater geboren wurde - der sei Anathe$m a{ }^{38}$ Daraufhin respondieren die Bischöfe im Chor mit dem bestätigenden Ruf anathema sit $!^{39}$ Dieselbe Abfolge, ein von Valens ausgerufenes Anathema und die kollektiv antwortenden Rufe der Bischöfe anathema sit, wiederholt sich sechsmal, ${ }^{40}$ bevor die Bischöfe gemeinsam mit den versammelten Massen Valens mit Applaus und Fußgetrampel (tripudium) unterbrechen. ${ }^{41}$ Die Freude der Bischöfe und der in der Kirche versammelten Öffentlichkeit findet also verbalen ebenso wie physischen Ausdruck. Hieronymus fand in den Akten darüber hinaus Lobeshymen auf Valens und Ausdrücke des Bedauerns darüber, ihn jemals als Häretiker verdächtigt zu haben, die er nicht im einzelnen wiedergibt. ${ }^{42}$

Hieronymus' Bericht unterstreicht, dass seine Darstellung anhand der Dokumente in öffentlichen und kirchlichen Archiven verifiziert werden könne und das Geschehen auch noch in lebendiger Erinnerung sei. Diese Akten, so beansprucht er, seien die Basis seines Berichts. ${ }^{43}$ Der bemerkenswerte Charakter der Ereignisse motiviert also eine besonders emphatische Betonung der Verlässlichkeit der Quellen, bzw. umgekehrt betrachtet: die Bedeutung, welche die Erzählung der Verlässlichkeit ihrer Quellen zumisst, zeigt an, dass das Geschehen als außergewöhnlich wahrgenommen wurde.

Die Freudenszenen werden von Bischof Claudius, der bereits für das anfängliche Verlesen zuständig war, wieder in eine Art geordneten Geschäftsgang zurückgeleitet. ${ }^{44}$ Die öffentliche Aufführung des Einvernehmens zwischen

38 Hier., c. Lucif. 18 (152,23-24 CANELlis [wie Anm. 25]): Si quis negat Christum Dominum, Dei filium, ante saecula ex Patre genitum, anathema sit!

39 Hier., c. Lucif. 18 (152,24-25 CANELlis [wie Anm. 25]): Ab uniuersis consonatum est: Anathema sit!

40 Hier., c.Lucif. 18 (152,25-154,34 CANellis [wie Anm. 25]). Zur Frage eines "zweiten" Betrugs von Rimini in den Formulierungen der Anathematismen, s. DuvaL, La «manœuvre frauduleuse» (wie Anm. 24), 98-103; LöHR, Die Entstehung (wie Anm. 19), 130-135. Eine ins Gegenteil gekehrte Interpretation eines möglichen Betrugs seitens der 'Nizäner' legt jüngst I. IMAGE, Nicene Fraud at the Council of Rimini, in: StPatr 62 (2013) 313-322, vor.

41 Hier., c. Lucif. 18 (154,33-36 CANELlis [wie Anm. 25]): In hoc uero, cuncti episcopi et tota simul ecclesia plausu quodam et tripudio Valentis uocem exceperunt.

42 Hier., c. Lucif. 18 (154,42f. CANellis [wie Anm. 25]).

43 Hier., c. Lucif. 18 (154,37-41 CANELLIS [wie Anm. 25]): Quod si quis a nobis fictum putauit, scrinia publica scrutetur. Plenae sunt certe Ecclesiarum chartae; recens adhuc rei memoria est. Supersunt homines qui illi synodo interfuerunt et, quod ueritatem firmet, ipsi Ariani haec ita ut diximus gesta non denegent. (156,51-53 CANELLIS [wie Anm. 25]): Quae si quis plenius discere cupit, in Ariminensis synodi actis reperiet, unde et nos ista libauimus.

44 Hier., c. Lucif. 18 (154,43f. CANELlis [wie Anm. 25]): ...idem Claudius qui supra legere coeperat, ait.... 
Valens und den Bischöfen hat ihr Ende noch nicht ganz erreicht. Nachdem sich die Versammlung ein wenig beruhigt hat, hebt Claudius hervor, dass Valens bei seinen Verurteilungen noch einige Punkte übersehen habe, die gleichfalls zu verwerfen seien. Nur noch ein Anathema wird ausgeführt, bevor Hieronymus summarisch auf weitere im gleichen Stil erfolgte Verwerfungen verweist. ${ }^{45}$ Zwar spricht Hieronymus dabei von der Verwerfung solcher Sätze durch Valens, ${ }^{46}$ aber das einzig ausgeschriebene Beispiel - und implizit auch sein zusammenfassender Bericht - deuten auf eine signifikante Veränderung im Ablaufmuster hin. Bischof Claudius bezeichnet nämlich in seinem Verfahrensvorschlag Valens nunmehr als Herrn und Bruder, behandelt ihn also als vollgültiges Synodenmitglied und impliziert bestehende Kirchengemeinschaft; er beantragt ausdrücklich weitere problematische Sätze gemeinschaftlich (in commune) zu verdammen. ${ }^{47}$ Tatsächlich ist auf die (von Hieronymus dem Protokoll entnommene) Anführung des ersten zu verwerfenden Satzes in dieser Sequenz nur notiert, dass alle (dixerunt cuncti) das Anathema aussprachen. Der Protokollauszug erwähnt nicht mehr, dass Valens zuerst und separat gesprochen habe wie zuvor. Selbst bei Hieronymus, dessen zusammenfassende Bemerkung ausschließlich Valens als denjenigen anführt, der solche Sätze verdammte, wird die tatsächliche Veränderung deutlich. Denn auch Hieronymus entnimmt dem Protokoll, dass die einzelnen Sätze jeweils von Claudius vorgetragen wurden - und eben nicht in der zuvor beschriebenen Wiese von Valens. Claudius formuliert sicher nicht spontan, sondern verliest höchstwahrscheinlich die Anathematismen aus einem vorbereiteten Dokument. Damit handelt er in der gleichen Funktion und Rolle, in der er eingangs einen kritikwürdigen Text verlesen hatte. ${ }^{48}$ Während Claudius also wiederum in der Rolle des Vorlesers agiert, hat sich die Rollenzuschreibung des Valens fundamental

45 Hier., c. Lucif. 18 (156,46-49 CANELLIs [wie Anm. 25]): Si quis dixerit Filium Dei esse quidem ante omnia saecula, sed non ante omne omnino tempus, ut ei aliquid anteferat, anathema sit! Dixerunt cuncti: Anathema sit!

46 Hier., c. Lucif. 18 (156,49-51 CANELLIS [wie Anm. 25]): Multaque alia quae suspiciosa uidebantur, ad pronuntiationem Claudii, Valens condemnauit.

47 Hier., c. Lucif. 18 (154,44-156,46 CANELlis [wie Anm. 25]): Sunt aliqua, quae subterfugerunt dominum et fratrem meum Valentem, quae, si uobis uidetur, ne qui scrupulus remaneat, in commune damnemus....

48 Dabei unterstreicht die Vorbereitung einer Liste von Anathematismen im übrigen nochmals den inszenierten Charakter des Geschehens; es läge nahe, auch Valens' frühere Interventionen in der Sitzung auf dieselbe vorbereitete Anathematismenliste zurückzuführen, und es wäre darüber hinaus durchaus vorstellbar, ja wahrscheinlich, dass die frühere Verwerfung arianischer "Blasphemien" vor dem Prätoriumspräfekten sich bereits auf diese Anathematismen bezieht. 
verändert. Nachdem die Synode kurz zuvor so demonstrativ ihre Freude über die Übereinstimmung zwischen Valens und den anderen Bischöfen ausagiert hatte, konnte er nun als vollständig integriert angesehen werden und mit allen anderen zusammen handeln. Claudius' Benutzung des Bruder-Titels veranschaulicht den gleichen Statuswechsel. Valens, der zunächst noch als unter Erklärungsnot stehender Einzelner im Gegenüber zum Kollektiv der Bischöfe sprach und auftrat, dessen Rolle also anfangs unsicher und angefochten war, handelt nun ganz und gar als vollwertiges Mitglied der Synode und geht völlig in der Gemeinschaft der Bischöfe auf.

Das Einvernehmen, wir dürfen sagen: der Friede, zwischen den Bischöfen ist in diesem Augenblick synodale und kirchliche Wirklichkeit. Im gemeinsamen Handeln als Bruder und Bruder, im gemeinsamen Anathema-Rufen, ist für die anwesende Öffentlichkeit ebenso wie für die Handelnden selbst der Friede wahrnehmbar und erlebbar. Nur so konnte der Makel heimlichtuerischen Aushandelns von theologischen Subtilitäten im kleinen Kreis und unter politischem Druck überwunden werden. Dem Verdacht, dass dabei etwas nicht mir rechten Dingen zugegangen war - die notorische fraus von Rimini - steht im scharfen Kontrast der öffentliche, vor aller Augen vollzogene, jubelnd und trampelnd ausagierte Friedensschluss gegenüber. Tripudium war ursprünglich eine Art Kriegstanz ${ }^{49}$ Zwar werden die Bischöfe wohl nicht in den Reihen getanzt haben, aber trampelnd und johlend dürfen wir sie uns vorstellen. So sah der synodale Friede von Rimini aus.

Zusammenfassend können wir ein komplexes Geflecht von theologischen, sozialen und symbolischen-performativen Reflexionen und Aktivitäten festhalten, die dem Wunsch nach kirchlichem Frieden im Kontext der Synoden des 4. Jh. Gestalt geben:

Die Arbeit an theologisch-sachlicher Übereinkunft, die in den Bekenntnissen und Formeln dieser Zeit so vielgestaltigen Ausdruck findet, beschreibt die notwendige Bedingung, unterschiedliche Ansichten und terminologische Präferenzen zu überwinden. Dabei ist das Bemühen um Klarheit und Präzision von der Sorge um ein Übermaß begrifflicher Subtilität und der Furcht vor dem resultierenden sozialen Konflikt- und Spaltungspotential der Sachdebatte stets begleitet. So ist die im Tomus ad Antiochenos gespiegelte Bereitschaft, den positiven Sinn einer abweichenden dogmatischen Terminologie anzuhören und ihre Intention als nicht häretisch anzuerkennen, auch wenn

49 Der von Hieronymus gewählte Ausdruck bezeichnet ursprünglich das rituelle Tanzen von Priestern, spezifisch das dem Kriegsgott Mars geweihte; s. OLD, sp. 1976 s.v. - Hieronymus kreiert so eine äußerst evokative Darstellung der Szene. 
man sie selbst nicht präferiert und ohne sie schon anzunehmen, ein wichtiger Durchbruch auf dem Weg zum Konsens in der Sache, auf dem allenfalls Frieden aufruhen konnte. Konfliktreiche persönliche Loyalitäten machten diese denkbare Basis eines Friedensschlusses letztendlich zunichte. Es ist deutlich, dass Übereinkunft nicht auf dem Papier allein zustande kommt; der Wille, die Vertreter anderer Gruppierungen nicht rundheraus zu verdächtigen, also in ein personales Verhältnis miteinander einzutreten, ist vielmehr ebenfalls gefordert. Dafür war im anderen hier skizzierten Fall des Valens von Mursa zusätzlich zu einem Konsenspapier - zunächst ein förmliches Dementi der Anhängerschaft von der Symbolgestalt der Häresie von Nöten. Die schon andauernde vorherige Streitgeschichte hatte aber eine gesellschaftliche Atmosphäre geschaffen, in der die Einigung zwischen Bischöfen, und das Gespräch mit zuständigen Reichsbeamten nicht ausreichend waren, um Frieden zu stiften. Diesem Defizit wirkt die Einbeziehung einer breiten kirchlichen Öffentlichkeit entgegen. Und dabei gewinnt eine performative Herangehensweise herausragende Bedeutung: Frieden, bischöfliche Gemeinschaft und Zusammenhalt müssen nach außen offen dargestellt, ja geradezu zelebriert werden. So wird die in den Anathematismen sachlich vollzogene Abweisung "falscher" Theologie aus der internen theologischen Fachdiskussion überführt in eine öffentliche Darstellung der in dieser Abgrenzung gewonnenen und anschaulichen werdenden gemeinsamen Identität. Wo die Synoden auf Frieden hinwirken und ihn, wie kurzlebig und angefochten auch immer, erreichen, sind all diese Aspekte erforderlich: theologische Sachklärung, Herstellung oder Heilung persönlichen Vertrauens und Suche nach gesellschaftlicher Akzeptanz in der performativen Zurschaustellung des auf beiden Feldern Erreichten als erlebbare Koinonia. Der auf der Synode hergestellte Friede bedarf der theologischen Übereinkunft, er braucht die nuancierten Formeln und Arbeitspapiere, er muss aber auch in die spätantike kirchliche Öffentlichkeit hinein sichtbar und hörbar vermittelt werden. 